\title{
Hypoxia and macrophages promote glioblastoma invasion by the CCL4-CCR5 axis
}

\author{
YING WANG ${ }^{1 *}$, TAO LIU ${ }^{2 *}$, NING YANG ${ }^{2,3}$, SHUO XU $^{2,3}$, XINGANG LI ${ }^{2,3}$ and DONGHAI WANG ${ }^{2,3}$ \\ ${ }^{1}$ Department of Rehabilitation, Qilu Children's Hospital of Shandong University, Jinan, Shandong; \\ ${ }^{2}$ Department of Neurosurgery, Qilu Hospital, Shandong University, Jinan, Shandong; \\ ${ }^{3}$ Brain Science Research Institute, Shandong University, Jinan, Shandong, P.R. China
}

Received April 4, 2016; Accepted August 16, 2016

DOI: $10.3892 / o r .2016 .5171$

\begin{abstract}
Glioblastoma (GBM) is a highly malignant brain tumor characterized by invasion tendency. Macrophage infiltration is associated with GBM invasion, but the mechanisms remain unclear. Hypoxia is an outstanding characteristic of GBM tissue. Hypoxia microenvironment modulates the biological behaviors of both tumor cells and infiltrated immune cells, including macrophages. In the present study, we analyzed the effects of hypoxia and macrophages on invasion of GBM cells and its potential mechanisms. We found that both hypoxia and macrophage supernatant promoted GBM cells invasion and matrix metalloproteinase (MMP)-9 expression, and hypoxia modulated the invasive activity of GBM cells by upregulating their CCR5 expression. The supernatant of hypoxic macrophages also showed greater pro-invasion effect than normoxic macrophages through the elevated secretion of CCL4. Moreover, we found that interferon regulatory factor-8 (IRF-8) was possibly involved in hypoxia-modulated CCL4 expression of macrophages. Taken together, the present study found that macrophages promoted GBM invasion by the CCL4-CCR5 axis, and hypoxia enhanced the interaction between these two types of cells by upregulating both CCL4 and CCR5 expression, respectively. The results of the present study suggested that hypoxia would be a potential target for the development of immune therapies of GBM.
\end{abstract}

Correspondence to: Dr Donghai Wang, Department of Neurosurgery, Qilu Hospital, Shandong University, 107 Wenhuaxi Road, Jinan, Shandong 250012, P.R. China

E-mail: donghai_w@126.com

${ }^{*}$ Contributed equally

Abbreviations: GBM, glioblastoma; CNS, central nervous system; TAM, tumor associated macrophage; MMP, matrix metalloproteinase; PMA, phorbol-12-myristate-13-acetate; NAb, neutralizing antibody; FBS, fetal bovine serum; IRF, interferon regulatory factor

Key words: hypoxia, macrophage, gliblastoma, invasion, CCL4, CCR5, IRF-8

\section{Introduction}

Glioblastoma (GBM) is the most common primary brain tumor characterized with high malignancy (1). Despite of the multiple therapies developed during the recent decades, including surgery, chemotherapy and radiation, the median survival of GBM is less than 16 months $(2,3)$. One of the reasons for such poor prognosis is the tendency of GBM to invade into surrounding brain parenchyma even at early phase of tumorigenesis (4). Infiltration of cancer cells into normal brain tissue makes it impossible for complete surgical removal, and increases the risk of resistance to conventional chemotherapy and radiation, finally leads to recurrence (5). Therefore, how to decrease the invasion activity of cancer cells became an important issue in the development of innovative strategies for GBM treatment.

Malignant cells are under the surveillance of immune cells to resist invasion, which is an important aspect of anticancer immunity. Although normal brain tissue is usually acknowledged as immunologically privileged, the impaired barrier of tumor vasculature of GBM facilitates the infiltration and accumulation of immune cells in the tumor microenvironment (6). Macrophages are the most abundant immune cells in GBM tissue, up to $30 \%$ of total tumor mass (7). GBM-infiltrated macrophages are composed by two different subgroups with distinct origin but similar phenotypes, including microglia arising from resident CNS macrophages, and tumor-associated macrophages arising form circulating monocytes $(8,9)$. These two subgroups are shown to promote GBM progression from multiple aspects, including vasculogenesis, immune suppression and GBM cell invasion $(10,11)$. Co-cultivation with primary microglia cells enhanced the expression and activation of matrix metalloproteinases (MMPs) of glioma cells, and also promoted their invasion into collagen matrix (12-14). However, the above studies only showed the invasion-promoting effects of microglia cells, and there is little evidence concerning the role of another major macrophage subgroup, the tumor-associated macrophages, in the modulation of GBM invasion process. Moreover, how macrophages adapted such phenotype and function in GBM microenvironment still needs further investigation.

Hypoxia is one of the well-acknowledged characteristics of GBM microenvironment, due to rapid cell proliferation and 
inadequate vascularization (15). Hypoxic GBM cells showed increased invasive capacity, resistance to therapies and poor prognosis of patients $(16,17)$. Besides tumor cells, hypoxia microenvironment also modulated the functions and biological behavior of immune cells infiltrated in the tumor, including macrophages. Previous studies have found that hypoxiamodulated macrophages promoted the invasive and metastatic behavior of various types of cancer cells, such as gastric and colon cancer (18-20). However, the effects of hypoxic tumor-associated macrophages on GBM invasion are largely unknown. Based on the above observations, we speculate that the hypoxia microenvironment in GBM may modulate the functions of tumor-associated macrophages, including their effects on GBM cell invasion. In the present study, we verified the above speculation by showing that hypoxia enhanced CCL4 expression in THP-1-derived macrophages and CCR5 expression in GBM cell line U87, respectively. The enhancement of CCL4-CCR5 axis led to increased MMP-9 expression in U87 cells, and promoted their invasive capacity. Our results suggested that hypoxia plays an important role in the development of a prone-invasion microenvironment in GBM tissue by not only directly affecting the biological behavior of tumor cells, but also modulating the functions of tumor-associated macrophages. Hypoxia in tumor tissue would be a potential target for the treatment of GBM.

\section{Materials and methods}

Reagents and antibodies. Phorbol-12-myristate-13-acetate (PMA) was obtained from Sigma-Aldrich (St. Louis, MO, USA). Monoclonal anti-human CCL4 neutralizing antibody (NAb) were obtained from R\&D Systems (Minneapolis, MN, USA). Fluorescein isothiocyanate (FITC) or phycoerythrin (PE)-labeled monoclonal antibodies for CCR2, CCR3, CCR4, CCR5 and CCR7 were obtained from R\&D Systems.

Cell culture and generation of THP-1-derived macrophages. Human GBM cell line U87 and acute monocytic leukemia cell line THP-1 was obtained from the American Type Culture Collection (ATCC; Manassas, VA, USA). The cells were cultured in RPMI-1640 medium supplemented with heatincubated $10 \%$ fetal bovine serum (FBS), $100 \mathrm{U} / \mathrm{ml}$ penicillin and $100 \mu \mathrm{g} / \mathrm{ml}$ streptomycin at $37^{\circ} \mathrm{C}$ in normoxic $\left(21 \% \mathrm{O}_{2}, 5 \%\right.$ $\left.\mathrm{CO}_{2}, 74 \% \mathrm{~N}_{2}\right)$ or hypoxic $\left(1 \% \mathrm{O}_{2}, 5 \% \mathrm{CO}_{2}, 95 \% \mathrm{~N}_{2}\right)$ incubators. In some cases, CCR5 siRNA or scramble control siRNA were transfected into U87 cells before the cultivation in normoxic or hypoxic conditions, and the details are described in the following section.

To generate THP-1-derived macrophages, $2 \times 10^{5}$ THP-1 cells were incubated in normoxic or hypoxic incubators and treated with $100 \mathrm{ng} / \mathrm{ml}$ PMA for $48 \mathrm{~h}$, as previously described (21). Then, the medium was washed and replaced by fresh RPMI1640 medium with $10 \%$ FBS for another $24 \mathrm{~h}$ under normoxic or hypoxic conditions to obtain macrophage supernatant.

Cultivation of U87 cells with macrophage supernatant. For the treatment of macrophage supernatant to GBM cells, $2 \times 10^{5}$ U87 cells in 2 ml RPMI-1640 medium with $10 \%$ FBS were plated into 6-well plate and incubated for $24 \mathrm{~h}$ under normoxic or hypoxic conditions. Then, the medium were replaced by $50 \%$ normoxic
Table I. Primer sequences for RT-PCR.

Gene name Primer sequences

\section{MMP-2 F 5'-CAA GTT TCC ATT CCG CTT C-3' R 5'-GTT CCC ACC AAC AGT GGA CA-3' \\ MMP-9 F 5'-TTG ACA GCG ACA AGA AGT GGG-3' R 5'-GCC ATT CAC GTC GTC CTT AT-3' \\ CCL3 F 5'-TGG CTC TCT GCA ACC AGT TCT-3' R 5'-GTA GCT GAA GCA GCA GGC G-3' \\ CCL4 F 5'-CCG TGT TAT TGT ATT AGG TG-3' R 5'-GAA TCA AAT GTG TTA TCC ATG T-3' \\ CCL5 F 5'-GGC AGC CCT CGC TGT CAT CCT CA-3' R 5'-CTT GAT GTG GGC ACG GGG CAG TG-3' \\ IRF-8 F 5'-AGT AGC ATG TAT CCA GGA CTG AT-3' \\ R 5'-CAC AGC GTA ACC TCG TCT TC-3' \\ GAPDH F 5'-GGT GGT CTC CTC TGA CTT CAA CAG-3' R 5'-GTT GCT GTA GCC AAA TTC GTT GT-3'}

MMP, matrix metalloproteinase; F, forward; R, reverse; IRF-8, interferon regulatory factor- 8 .

or hypoxic macrophage supernatant, respectively, and cultured for another $24 \mathrm{~h}$ under normoxic or hypoxic conditions. After the cultivation procedure, U87 cells were collected for further invasion assay. In some cases, CCL4 (NAb, $50 \mu \mathrm{g} / \mathrm{ml})$ was added along with macrophage supernatant.

Cell invasion assay. The invasion assay of U87 cells was performed as previously described (22) with minor modifications. Briefly, $50 \mu \mathrm{l}$ of diluted 1:4 Matrigel in serum-free RPMI-1640 medium was added to the upper chamber of 24-well Transwell inserts $(8-\mu \mathrm{m}$ pores; both from BD Biosciences, Franklin Lakes, NJ, USA) and incubated at $37^{\circ} \mathrm{C}$ overnight for gelling. U87 cells $\left(5 \times 10^{4}\right)$, previously treated with/without macrophage supernatant for $24 \mathrm{~h}$, were resuspended in $100 \mu \mathrm{l}$ serum-free medium and were plated into the upper chamber of Transwell inserts coated with Matrigel. RPMI-1640 medium $(600 \mu \mathrm{l})$ containing $10 \%$ FBS were added to the lower chamber. After incubating under normoxic or hypoxic conditions for $48 \mathrm{~h}$, the noninvaded cells on top of the Transwell were removed with a cotton swab. The invaded cells were fixed by $10 \%$ formalin and stained with eosin. Three fields of each well were photographed, and cell numbers were determined.

Real-time quantitative RT-PCR. Total RNA was extracted by TRIzol reagent (Invitrogen, Carlsbad, CA, USA), and cDNA was synthesized through the reverse transcription. Total RNA $(1.0 \mu \mathrm{g})$ was transcribed into cDNA with oligo(dT)16 primers and Moloney murine leukemia virus reverse transcriptase according to the manufacturer's instructions (Invitrogen). Quantitative RT-PCR was performed on the LightCycler 2.0 Instrument (Roche Diagnostic, Mannheim, Germany). GAPDH was used as an internal control. Primers for MMP-2, MMP-9, CCL3, CCL4, CCL5, interferon regulatory factor-8 (IRF-8) and GAPDH are listed in Table I. Five microliters 
A

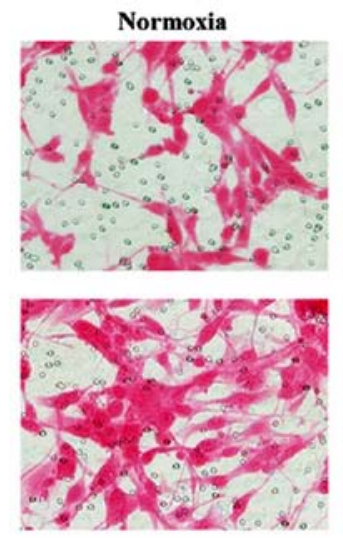

B

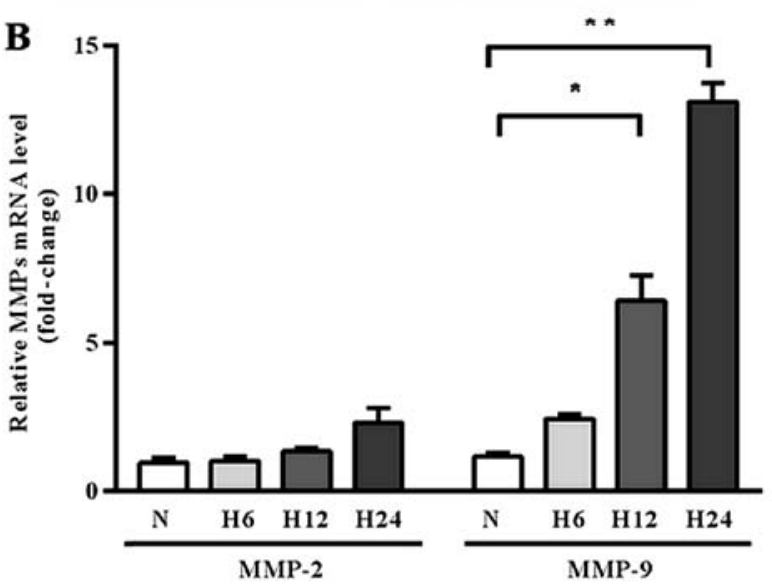

Hypoxia

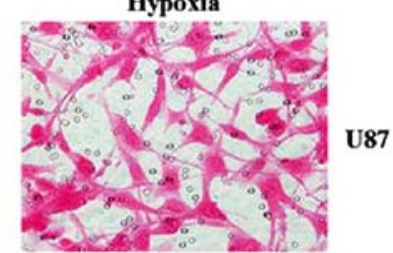

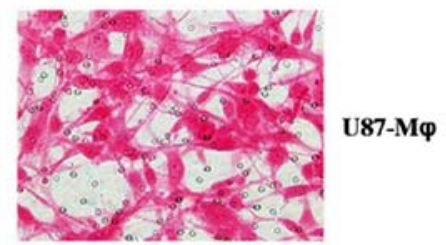

C

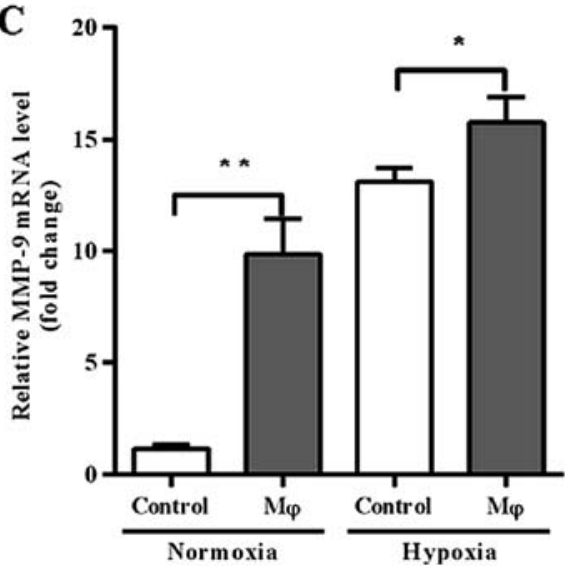

Figure 1. Hypoxia and macrophage supernatant promote MMP-9 expression and invasion of U87 cells. (A) U87 cells were treated in normoxia or hypoxia conditions with/without supernatant of normoxic or hypoxic macrophage stimulation, and then invasion assay was performed. Number of invasive cells was the mean value of five random fields under a light microscope. Original magnification, x400. (B) The transcription of MMP-2 and MMP-9 under the treatment of different period time of hypoxia. (C) The transcription of MMP-9 under the treatment of hypoxia and macrophage (M $\phi$ ) supernatant. The RT-PCR data were normalized to the control and shown as the fold-change. Each bar represents the mean $\pm \operatorname{SD}\left(n=3 ;{ }^{*} p<0.05,{ }^{* *} p<0.01\right)$.

of $2 \mathrm{X} \mathrm{SYBR}^{\circledR}$-Green qPCR Master mix (Thermo Fisher Scientific, Waltham, MA, USA), $1 \mu \mathrm{l}$ of forward primer, $1 \mu \mathrm{l}$ of reverse primer, $1 \mu \mathrm{l}$ of cDNA and $2 \mu \mathrm{l}$ of $\mathrm{ddH}_{2} \mathrm{O}$ was added into the reaction mixture and incubated at $94^{\circ} \mathrm{C}$ for $30 \mathrm{sec}$, $58^{\circ} \mathrm{C}$ for $30 \mathrm{sec}$ and $72^{\circ} \mathrm{C}$ for $45 \mathrm{sec}$ for 40 cycles. The mRNA level of each sample was measured by the $2^{-\Delta \Delta C t}$ method (23).

Flow cytometry. To analyze the expression of chemokine receptors CCR2, CCR3, CCR4, CCR5 and CCR7, normoxic or hypoxic U87 cells were stained by monoclonal antibodies labeled with FITC or PE. Isotype controls were run in parallel. The analysis was performed by a FACSCalibur flow cytometer, and the mean fluorescence intensity was determined by CellQuest version 3.3 software (BD Biosciences).

RNA interference. Small-interference RNA (siRNA) targeting CCR5 and scramble control siRNA were designed by GenePharma Co., Ltd (Shanghai, China). For transient silencing, $5 \times 10^{4} / \mathrm{ml}$ U87 cells were seeded onto 24 -well plates and transfected with siRNA (all $80 \mathrm{nmol} / \mathrm{l}$ ) using Lipofectamine RNAiMAX reagent (Invitrogen) following the manufacturer's protocol.

Enzyme-linked immunosorbent assay (ELISA). The supernatant of normoxic or hypoxic THP-1-derived macrophages was collected. The concentrations of CCL3, CCL4 and CCL5 in supernatant were determined by ELISA kits (R\&D Systems) according to the manufacturer's instructions.

Statistical analysis. Data are primarily presented as the mean \pm SD. The SPSS software package (version 13.0; SPSS, Inc., Chicago, IL, USA) was used for all statistical analysis. The distribution of the samples was determined via Kolmogorov-Smirnov test. The results of experiments were analyzed by t-test or one-way ANOVA wherever appropriate. Tukey post hoc comparison was performed when statistical significance $(\mathrm{p}<0.05)$ was found between observations.

\section{Results}

Hypoxia and macrophage supernatant promote invasion of U87 cells by upregulating MMP-9 expression. Firstly, we analyzed the effects of hypoxia and macrophage supernatant on invasion of GBM cell line U87 by Matrigel-coated Transwell assay. The results showed that both hypoxia and macrophage supernatant promoted U87 invasion. Moreover, U87 cells gained more invasive capacity when they were pre-treated by hypoxic macrophage supernatant before cultured under hypoxia conditions (Fig. 1A).

We further investigated whether the modification of U87 cell invasive activity was dependent on MMPs expression, since they are acknowledged for the involvement in cell invasion 
A
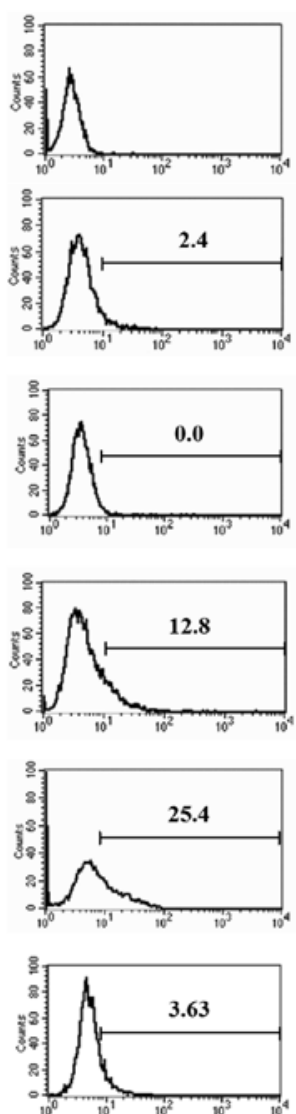
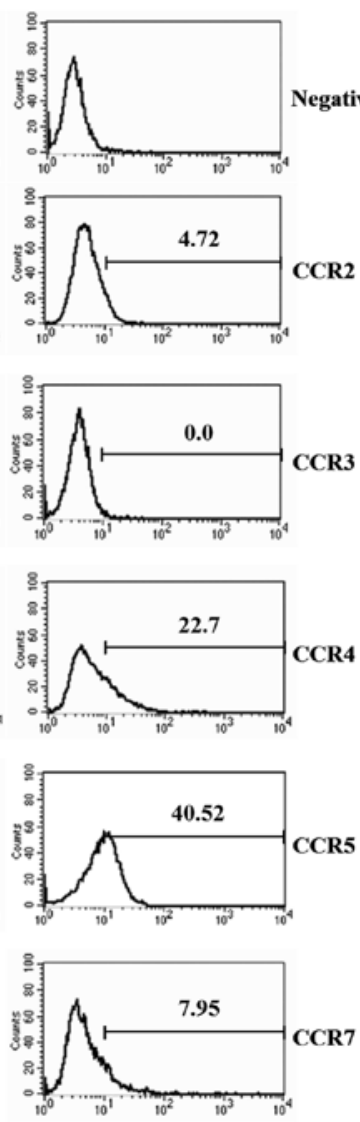

B

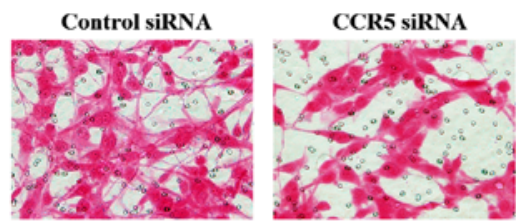

Number of invaded cells
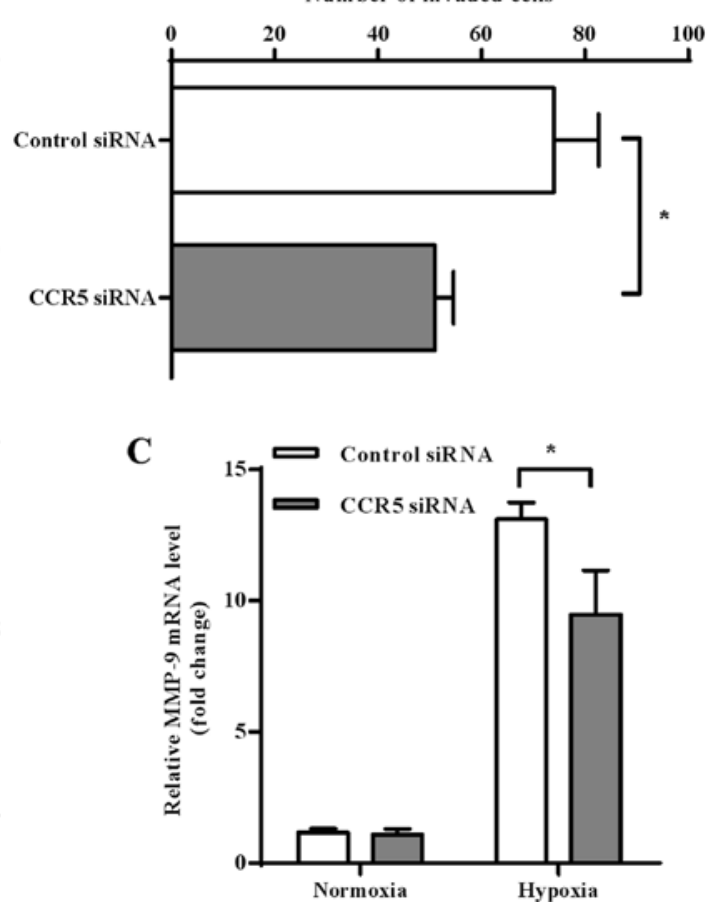

Figure 2. Hypoxia promotes MMP-9 expression and invasion of U87 cells by upregulating CCR5. (A) The expression of CCR2, CCR3, CCR4, CCR5 and CCR7 on U87 cells was determined by flow cytometry. (B) The number of invasive U87 cells in hypoxia with/without CCR5 siRNA transfection. Number of invasive cells was the mean value of five random fields under a light microscope. Original magnification, $\mathrm{x} 400$. (C) The transcription of MMP-9 under the treatment of normoxia or hypoxia with/without CCR5 siRNA transfection. The RT-PCR data were normalized to the control and shown as the fold-change. Each bar represents the mean $\pm \mathrm{SD}\left(\mathrm{n}=3,{ }^{*} \mathrm{p}<0.05\right)$.

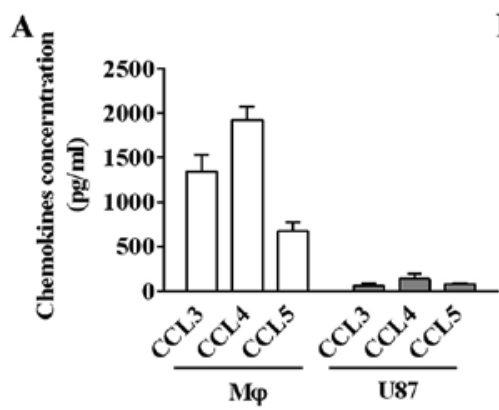

D

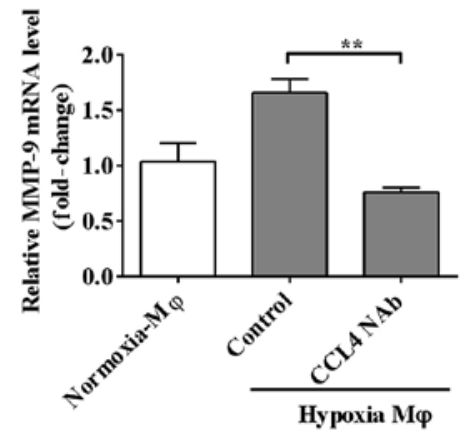

B

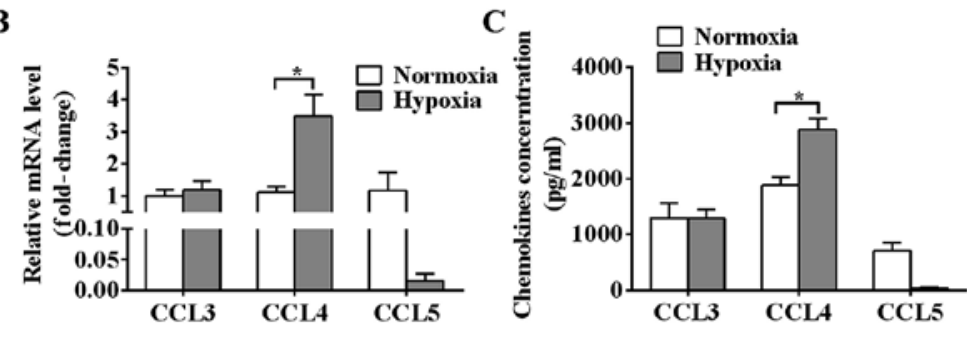

$\mathbf{E}$

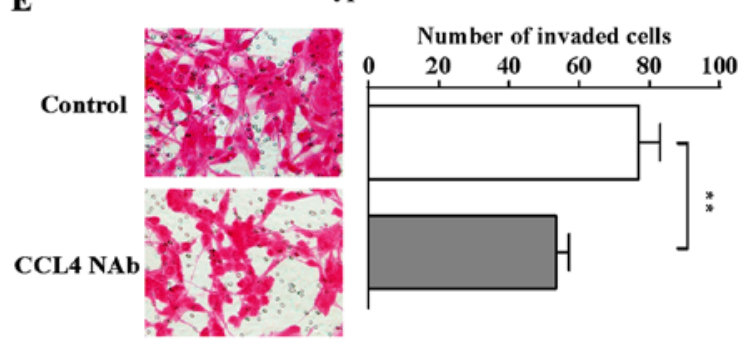

Figure 3. Hypoxia enhances CCL4 secretion in macrophages (M $\phi$ ) involved in MMP-9 expression and invasion of U87 cells. (A) The secretion of CCL3, CCL4 and CCL5 of THP-1-derived macrophages and U87 cells were measured by ELISA. (B and C) The transcription (B) and secretion (C) of CCL3, CCL4 and CCL5 of THP-1-derived macrophages in normoxia or hypoxia were determined. (D) CCL4 NAb was added along with the hypoxic macrophage supernatant into the hypoxic U87 cells. Normoxic macrophage supernatant without CCL4 NAb was used as a negative control. The transcription of MMP-9 of U87 cells was analyzed. (E) Hypoxic U87 cells, treated by hypoxic macrophage supernatant with/without CCL4 NAb were analyzed in invasion assay. The number of invasive cells was counted. Each bar represents the mean $\pm \mathrm{SD}\left(\mathrm{n}=3 ;{ }^{*} \mathrm{p}<0.05,{ }^{* *} \mathrm{p}<0.01\right)$. 

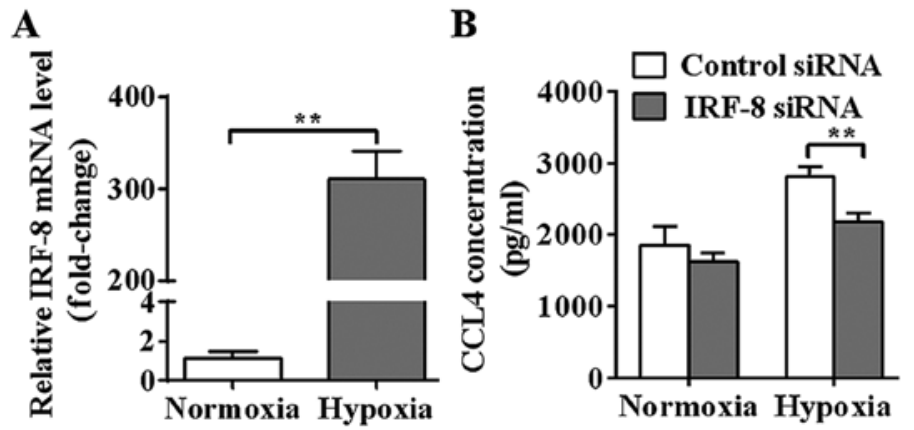

Figure 4. Hypoxia promotes CCL4 secretion of macrophages by upregulating IRF-8. (A) The transcription of IRF-8 in normoxic or hypoxic THP-1-derived macrophages was analyzed. The RT-PCR data were normalized to the control and shown as the fold-change. (B) The secretion of CCL4 in normoxic or hypoxic macrophages with/without IRF-8 siRNA transfection was determined by ELISA. Each bar represents the mean $\pm \operatorname{SD}\left(n=3 ;{ }^{* *} \mathrm{p}<0.01\right)$.

process. We analyzed the expression of MMP-2 and MMP-9 in U87 cells, which are two of the most studied MMPs and closely associated with cell invasive capacity. Firstly, we explored the effect of hypoxia on MMP-2 and MMP-9 expression at different time point. As shown in Fig. 1B, the expression of MMP-2 in U87 cells remained relatively constant in hypoxic condition, but MMP-9 was significantly upregulated under hypoxia stimulation in a time-related fashion $(\mathrm{p}=0.0108$ at $12 \mathrm{~h}, \mathrm{p}=0.0006$ at $24 \mathrm{~h}$ ). Secondly, we analyzed the expression of MMP-9 under the treatment of macrophage supernatant and found that macrophage supernatant upregulated MMP-9 expression either in normoxic or hypoxic conditions (Fig. 1C; $\mathrm{p}=0.0114$ and 0.0118 , respectively).

Hypoxia increases CCR5 expression regulated MMP-9 expression and invasion of U87 cells. Chemokine receptors have been shown as important regulators for invasion process of cancer cells (21). In order to investigate whether the axis of chemokine-chemokine receptors was involved in hypoxia-modulated invasion of GBM cells, we measured expression of several CC chemokine receptors (CCRs) on normoxic and hypoxic U87 cells. The results of flow cytometry found relatively high expression of CCR4 and CCR5 on U87 cells, while low expression of CCR 2 and CCR7 and no CCR3 was found. Both CCR4 and CCR5 expression was significantly increased after hypoxia treatment, but CCR5 showed higher expression than CCR4 in both normoxic and hypoxic U87 cells (Fig. 2A).

Based on the above observations, we chose CCR5 for further investigation into its possible role in GBM invasion. We knocked down CCR5 expression on U87 cells by siRNA transfection and found that CCR5 downregulation significantly decreased invasion activity of hypoxic U87 cells (Fig. 2B; $\mathrm{p}=0.0237$ ). We also found that CCR5 knockdown decreased MMP-9 transcription in hypoxic U87 cells $(\mathrm{p}=0.0100)$, while no significant downregulation was observed under normoxia condition (Fig. 2C).

Hypoxia increases CCL4 secretion in THP-1-derived macrophage-regulated MMP-9 expression and U87 invasion. Macrophages are important targets and sources of chemokines. Since the above results found that hypoxic macrophage supernatant promoted U87 cells invasion, and hypoxia upregulated CCR5 expression affected the invasion process, we further investigated whether CCR5-related chemokines, including CCL3, CCL4 and CCL5 in hypoxic macrophage supernatant were involved in this modulation. As showed by our results, the concentrations of CCR5-related chemokines secreted by THP-1-derived macrophages were higher than those secreted by U87 cells (Fig. 3A), and they exhibited different expression profile under hypoxia stimulation. Only the expression of CCL4 was significantly upregulated $(\mathrm{p}=0.0149)$ in hypoxic THP-1derived macrophages, while CCL5 was decreased and CCL3 remained relatively unchanged (Fig. 3B). The results of ELISA were consistent with RT-PCR results (Fig. 3C; $\mathrm{p}=0.0385$ ).

In order to verify whether upregulated CCL4 secreted by hypoxic macrophages promoted U87 invasion process, CCL4 $\mathrm{NAb}(50 \mu \mathrm{g} / \mathrm{ml})$ was added into hypoxic macrophage supernatant before its stimulation toward U87 cells. We found that CCL4 NAb in hypoxic macrophage supernatant significantly inhibited both MMP-9 expression and invasion capacity of U87 cells (Fig. 3D and E; $\mathrm{p}=0.0086$ and 0.0032 , respectively).

Hypoxia promotes IRF8 involved in CCL4 expression in THP-1-derived macrophages. We went on to explore the mechanism by which hypoxia regulated CCL4 expression in THP-1-derived macrophages. Interferon regulatory factors (IRFs) are important transcription factors in the maturation and differentiation of myeloid cells, and are closely associated with the function of macrophages. We first investigated whether hypoxia may regulate expression of IRFs in THP-1derived macrophages, and surprisingly found that hypoxia greatly increased IRF- 8 transcription in macrophages by $\sim 300$ times (Fig. 4A). To verify whether increased IRF-8 was involved in CCL4 expression of THP-1-derived macrophages, we knocked down its transcription by siRNA transfection. As Fig. 4B shows IRF-8 downregulation significantly decreased CCL4 secretion of hypoxic THP-1-derived macrophages $(\mathrm{p}=0.0053)$, while had little effects on normoxic CCL4 secretion $(\mathrm{p}=0.2396)$.

\section{Discussion}

In the present study, we analyzed the effects of macrophage supernatant on invasive capacity of glioblastoma (GBM) cells, and the modulation of hypoxia in this process. We found that THP-1-derived macrophage-secreted CCL4 promoted MMP-9 expression on U87 cells by interacting with CCR5. 
Hypoxia enhanced CCL4 secretion in THP-1-derived macrophages by upregulating the transcriptional factor IRF8, and also promoted CCR5 expression on GBM U87 cells, therefore promoting the interaction between these two cell lines. The enhanced CCL4-CCR5 axis further increased the invasive activities and MMP-9 transcription of U87 cells. The results of the present study revealed the effects of hypoxia on the interactions and possible mechanisms that occurred between macrophages and GBM cells, and the role of this interaction on GBM invasion. The present study also suggested that hypoxia microenvironment may be a potential target for resisting the invasion in the treatment of GBM.

The axis of chemokines and chemokine receptors has been demonstrated as important regulators in the invasion and metastasis process of cancer cells. CCR5, whose ligands include CCL3 (MIP-1 $\alpha$ ), CCL4 (MIP-1 $\beta$ ) and CCL5 (RANTES), was reported to be highly expressed in GBM tissue $(24,25)$. A recent study showed that the overexpression of CCR5 was associated with poor prognosis of GBM patients, and activation of CCR5 signaling promoted the invasion of U87 and U251 cell lines in vitro by upregulating MMP-9 expression (24). These observations were consistent with our findings, as we found that CCR5 knockdown in U87 cells reduced their invasive capacity as well as MMP-9 transcription. However, until now there are few clues to explain the formation of such CCR5 overexpressed characteristics occurring in GBM tissue. In the present study, we occasionally found that hypoxia significantly upregulated CCR5 expression in U87 cells. The upregulation of hypoxia on CCR5 expression was also found in breast cancer cells by a series of transcriptional and post-transcriptional modulations (26). Taken together, these results suggested that hypoxia may be a common regulator for CCR5 expression in tumor microenvironment.

Macrophages are important sources of chemokines. According to our results, THP-1-derived macrophages expressed relatively high concentration of all three CCR5associated chemokines, including CCL3, CCL4 and CCL5, whereas the expression of these chemokines was low in U87 cells. Hence, we speculated that macrophage-derived chemokines may at least partially explain why macrophage supernatant could promote GBM cell invasion, and why hypoxia supernatant had higher promoting effects. In the present study, we found that CCL4 was the only one that was promoted by hypoxia in all three CCR5-associated chemokines produced by THP-1-derived macrophages, and its expression in supernatant enhanced the invasion and MMP-9 expression of U87 cells. Several chemokine/chemokine receptors have been shown to promote an invasive phenotype in glioma cells. One of the most acknowledged was the stromal cell-derived factor-1 $\alpha$ (SDF-1 $\alpha$ )-CXCR4 system (27). The CXCR4 expression in glioma cell lines was upregulated under hypoxia stimulation, and enhanced their migrating or invasive activities in respond to SDF-1 $\alpha$ (28). Our studies found a new axis, the CCL4-CCR5 system, which played key roles in hypoxic GBM cell invasion. As macrophages were one of the major CCL4 sources in GBM tissue, this axis also emerged as important mediator in the interactions between tumorassociated macrophages and GBM cells, and hypoxia may enhance this interaction by promoting both CCL4 and CCR5 expression in the two cell types, respectively.
The mechanism by which hypoxia promoted CCL4 expression in macrophages was also preliminarily explored in the present study. Interferon regulatory factors (IRFs) are an important family of intracellular protein that regulate maturation and polarization of macrophages (29). Various members of this family, such as IRF-1 and IRF-3, have been shown to induce chemokines expression in macrophages (30-32). In the present study, we found for the first time that hypoxia greatly enhanced the transcription of IRF-8 in THP-1-derived macrophages, and IRF-8 was involved in CCL4 expression. Previous studies found that IRF-8 plays a dominant role not only in the differentiation of macrophages from their immature progenitors, but also in their phenotypes and functions. For example, IRF-8 induces a M1-type gene profile upon TLR stimulation, enhancing the expression of a number of pro-inflammatory cytokines, including IFN- $\beta$, IL-12p40 and IL-12p35 (33). Our findings provided more evidence on the modulation of IRF-8 in chemokines secretion of macrophage. These findings will help to refine the roles of IRF-8 in various physiological or pathological processes, such as tumor development, inflammation and tissue repair, since all these processes are characterized with change of oxygen tension and infiltration of immunocytes, particularly macrophages. However, how hypoxia modulated IRF-8 expression in macrophages was not demonstrated in the present study. As the interaction between IRF-8 and chromatin is repressed by small ubiquitin-like modifiers (SUMO), and hypoxia is an important regulator of sumoylation status in variant types of cells (34-36), the post-transcriptional modulation of IRF-8 could be an important mechanisms in hypoxic macrophages.

In conclusion, the present study suggested for the first time that macrophage-secreted CCL4 would promote the invasive capacity of GBM cells by interacting with CCR5 receptor, and hypoxia enhanced this interaction by upregulating both CCL4 and CCR5 expression in these two cell types. Our findings will define additional roles of tumor-infiltrated macrophages in GBM development, and contributed to better understanding of how tumor hypoxia microenvironment modifies local immune system in the pathophysiology of GBM.

\section{Acknowledgements}

The present study was supported by the Foundation of Shandong Provincial Science and Technology Development Plan (2014GGH218017) and the Special Foundation for Taishan Scholars (no. ts20110814).

\section{References}

1. Huse JT and Holland EC: Targeting brain cancer: Advances in the molecular pathology of malignant glioma and medulloblastoma. Nat Rev Cancer 10: 319-331, 2010.

2. Davis FG, McCarthy BJ, Freels S, Kupelian V and Bondy ML: The conditional probability of survival of patients with primary malignant brain tumors: Surveillance, epidemiology, and end results (SEER) data. Cancer 85: 485-491, 1999.

3. Meyer MA: Malignant gliomas in adults. N Engl J Med 359: 1850,2008

4. Kanu OO, Mehta A, Di C, Lin N, Bortoff K, Bigner DD, Yan H and Adamson DC: Glioblastoma multiforme: A review of therapeutic targets. Expert Opin Ther Targets 13: 701-718, 2009.

5. Giese A, Bjerkvig R, Berens ME and Westphal M: Cost of migration: Invasion of malignant gliomas and implications for treatment. J Clin Oncol 21: 1624-1636, 2003. 
6. Hussain SF, Yang D, Suki D, Aldape K, Grimm E and Heimberger AB: The role of human glioma-infiltrating microglia/macrophages in mediating antitumor immune responses. Neuro Oncol 8: 261-279, 2006.

7. Graeber MB, Scheithauer BW and Kreutzberg GW: Microglia in brain tumors. Glia 40: 252-259, 2002.

8. Kushchayev SV, Kushchayeva YS, Wiener PC, Scheck AC, Badie B and Preul MC: Monocyte-derived cells of the brain and malignant gliomas: The double face of Janus. World Neurosurg 82: 1171-1186, 2014.

9. Yang I, Han SJ, Kaur G, Crane C and Parsa AT: The role of microglia in central nervous system immunity and glioma immunology. J Clin Neurosci 17: 6-10, 2010.

10. Charles NA, Holland EC, Gilbertson R, Glass R and Kettenmann H: The brain tumor microenvironment. Glia 60 502-514, 2012.

11. da Fonseca AC and Badie B: Microglia and macrophages in malignant gliomas: Recent discoveries and implications for promising therapies. Clin Dev Immunol 2013: 264124, 2013.

12. Ye XZ, Xu SL, Xin YH, Yu SC, Ping YF, ChenL, Xiao HL, Wang B, Yi L, Wang QL, et al: Tumor-associated microglia/macrophages enhance the invasion of glioma stem-like cells via TGF- $\beta 1$ signaling pathway. J Immunol 189: 444-453, 2012.

13. Markovic DS, Vinnakota K, Chirasani S, Synowitz M, Raguet H, Stock K, Sliwa M, Lehmann S, Kälin R, van Rooijen N, et al: Gliomas induce and exploit microglial MT1-MMP expression for tumor expansion. Proc Natl Acad Sci USA 106: 12530-12535, 2009.

14. Lin HC, Song TY and Hu ML: S-Adenosylhomocysteine promotes the invasion of C6 glioma cells via increased secretion of matrix metalloproteinase-2 in murine microglial BV2 cells. Toxicol Sci 112: 322-330, 2009.

15. Evans SM, Judy KD, Dunphy I, Jenkins WT, Hwang WT, Nelson PT, Lustig RA, Jenkins K, Magarelli DP, Hahn SM, et al: Hypoxia is important in the biology and aggression of human glial brain tumors. Clin Cancer Res 10: 8177-8184, 2004

16. Kessler J, Hahnel A, Wichmann H, Rot S, Kappler M, Bache M and Vordermark D: HIF-1 $\alpha$ inhibition by siRNA or chetomin in human malignant glioma cells: Effects on hypoxic radioresistance and monitoring via CA9 expression. BMC Cancer 10 $605,2010$.

17. Yang L, Lin C, Wang L, Guo H and Wang X: Hypoxia and hypoxia-inducible factors in glioblastoma multiforme progression and therapeutic implications. Exp Cell Res 318: 2417-2426, 2012.

18. Shen Z, Kauttu T, Seppänen H, Vainionpää S, Ye Y, Wang S, Mustonen $\mathrm{H}$ and Puolakkainen P: Both macrophages and hypoxia play critical role in regulating invasion of gastric cancer in vitro. Acta Oncol 52: 852-860, 2013.

19. Shen Z, Seppänen H, Vainionpää S, Ye Y, Wang S, Mustonen $H$ and Puolakkainen P: IL10, IL11, IL18 are differently expressed in $\mathrm{CD} 14^{+}$TAMs and play different role in regulating the invasion of gastric cancer cells under hypoxia. Cytokine 59: 352-357, 2012.

20. Mu L, Wang J, Chen Y, Li L, Guo X, Zheng S and Jing C: Hypoxia-inducible factor-1 $\alpha$ and semaphorin4D genes involved with tumor-associated macrophage-induced metastatic behavior and clinical significance in colon cancer. Chin Med J 127: 3568-3575, 2014

21. Zhao P, Gao D, Wang Q, Song B, Shao Q, Sun J, Ji C, Li X, Li P and Qu X: Response gene to complement 32 (RGC-32) expression on M2-polarized and tumor-associated macrophages is M-CSF-dependent and enhanced by tumor-derived IL-4. Cell Mol Immunol 12: 692-699, 2015.
22. Chen S, Han M, Chen W, He Y, Huang B, Zhao P, Huang Q, Gao L, Qu X and Li X: KIF1B promotes glioma migration and invasion via cell surface localization of MT1-MMP. Oncol Rep 35: 971-977, 2016.

23. Livak KJ and Schmittgen TD: Analysis of relative gene expression data using real-time quantitative PCR and the $2^{-\Delta \Delta C_{\mathrm{T}}}$ method. Methods 25: 402-408, 2001.

24. Zhao L, Wang Y, Xue Y, Lv W, Zhang Y and He S: Critical roles of chemokine receptor CCR5 in regulating glioblastoma proliferation and invasion. Acta Biochim Biophys Sin 47: 890-898, 2015.

25. Pham K, Luo D, Liu C and Harrison JK: CCL5, CCR1 and CCR5 in murine glioblastoma: Immune cell infiltration and survival rates are not dependent on individual expression of either CCR1 or CCR5. J Neuroimmunol 246: 10-17, 2012.

26. Lin S, Wan S, Sun L, Hu J, Fang D, Zhao R, Yuan S and Zhang L: Chemokine $\mathrm{C}-\mathrm{C}$ motif receptor 5 and $\mathrm{C}-\mathrm{C}$ motif ligand 5 promote cancer cell migration under hypoxia. Cancer Sci 103: 904-912, 2012.

27. Zhang J, Sarkar S, Cua R, Zhou Y, Hader W and Yong VW: A dialog between glioma and microglia that promotes tumor invasiveness through the CCL2/CCR2/interleukin-6 axis. Carcinogenesis 33: 312-319, 2012.

28. Zagzag D, Lukyanov Y, Lan L, Ali MA, Esencay M, Mendez O, Yee H, Voura EB and Newcomb EW: Hypoxia-inducible factor 1 and VEGF upregulate CXCR4 in glioblastoma: Implications for angiogenesis and glioma cell invasion. Lab Invest 86: 1221-1232, 2006.

29. Günthner R and Anders HJ: Interferon-regulatory factors determine macrophage phenotype polarization. Mediators Inflamm 2013: 731023, 2013

30. Génin P, Algarté M, Roof P, Lin R and Hiscott J: Regulation of RANTES chemokine gene expression requires cooperativity between NF-kappa B and IFN-regulatory factor transcription factors. J Immunol 164: 5352-5361, 2000

31. Liu J, Guan X and Ma X: Interferon regulatory factor 1 is an essential and direct transcriptional activator for interferon $\gamma$-induced RANTES/CCl5 expression in macrophages. J Biol Chem 280: 24347-24355, 2005.

32. Yarilina A, Park-Min KH, Antoniv T, Hu X and Ivashkiv LB: TNF activates an IRF1-dependent autocrine loop leading to sustained expression of chemokines and STAT1-dependent type I interferon-response genes. Nat Immunol 9: 378-387, 2008.

33. Holtschke T, Löhler J, Kanno Y, Fehr T, Giese N, Rosenbauer F, Lou J, Knobeloch KP, Gabriele L, Waring JF, et al: Immunodeficiency and chronic myelogenous leukemia-like syndrome in mice with a targeted mutation of the ICSBP gene. Cell 87: 307-317, 1996.

34. Koh MY, Nguyen V, Lemos R Jr, Darnay BG, Kiriakova G, Abdelmelek M, Ho TH, Karam J, Monzon FA, Jonasch E, et al: Hypoxia-induced SUMOylation of E3 ligase HAF determines specific activation of HIF2 in clear-cell renal cell carcinoma. Cancer Res 75: 316-329, 2015.

35. Sun L, Li H, Chen J, Iwasaki Y, Kubota T, Matsuoka M, Shen A, Chen $\mathrm{Q}$ and Xu Y: PIASy mediates hypoxia-induced SIRT1 transcriptional repression and epithelial-to-mesenchymal transition in ovarian cancer cells. J Cell Sci 126: 3939-3947, 2013.

36. Wu YC, Ling TY, Lu SH, Kuo HC, Ho HN, Yeh SD, Shen CN and Huang YH: Chemotherapeutic sensitivity of testicular germ cell tumors under hypoxic conditions is negatively regulated by SENP1-controlled sumoylation of OCT4. Cancer Res 72: 4963-4973, 2012. 